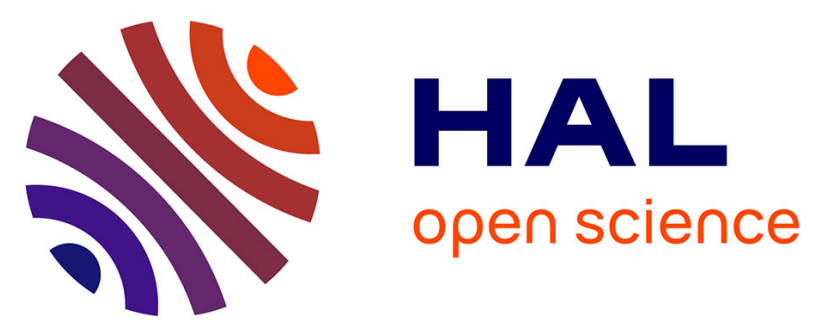

\title{
Advanced synthesis of highly crystallized hexagonal boron nitride by coupling polymer-derived ceramics and spark plasma sintering processes-influence of the crystallization promoter and sintering temperature \\ Yangdi Li, Vincent Garnier, Catherine Journet, Julien Barjon, Annick Loiseau, Ingrid Stenger, Alexandre Plaud, Berangere Toury, Philippe Steyer
}

\section{To cite this version:}

Yangdi Li, Vincent Garnier, Catherine Journet, Julien Barjon, Annick Loiseau, et al.. Advanced synthesis of highly crystallized hexagonal boron nitride by coupling polymer-derived ceramics and spark plasma sintering processes-influence of the crystallization promoter and sintering temperature. Nanotechnology, 2019, 30 (3), pp.035604. 10.1088/1361-6528/aaebb4 . hal-02063822

\author{
HAL Id: hal-02063822 \\ https://hal.science/hal-02063822
}

Submitted on 13 Apr 2021

HAL is a multi-disciplinary open access archive for the deposit and dissemination of scientific research documents, whether they are published or not. The documents may come from teaching and research institutions in France or abroad, or from public or private research centers.
L'archive ouverte pluridisciplinaire HAL, est destinée au dépôt et à la diffusion de documents scientifiques de niveau recherche, publiés ou non, émanant des établissements d'enseignement et de recherche français ou étrangers, des laboratoires publics ou privés. 


\title{
Advanced synthesis of highly-crystallized hexagonal boron nitride by coupling PDCs and SPS processes - influence of the crystallization promoter and sintering temperature
}

Yangdi Li ${ }^{1,2}$, Vincent Garnier ${ }^{1}$, Catherine Journet ${ }^{2}$, Julien Barjon ${ }^{3}$, Annick Loiseau ${ }^{4}$, Ingrid Stenger $^{3}$, Alexandre Plaud ${ }^{3}$, Bérangère Toury ${ }^{2}$, Philippe Steyer $^{1 *}$

1. Univ. Lyon, INSA-Lyon, MATEIS, UMR CNRS 5510, 7 Avenue Jean Capelle, F-69621, Villeurbanne, France

2. Univ. Lyon, Université Lyon 1, LMI, UMR CNRS 5615, 6 rue Victor Grignard, F-69622, Villeurbanne, France

3. Groupe d'Etude de la Matière Condensée, Université Versailles St Quentin \& CNRS, 45 Avenue des Etats-Unis, F-78035 Versailles, France

4. Laboratoire d'Étude des Microstructures (LEM), CNRS, ONERA, Université Paris-Saclay, 29 Avenue de la Division Leclerc, F-92322, Châtillon, France

* E-mail : philippe.steyer@insa-lyon.fr

\begin{abstract}
Hexagonal boron nitride nanosheets (BNNSs) are promising 2D materials for their exceptional chemical and thermal stabilities together with electrical insulating properties. A combined synthesis method involving the Polymer Derived Ceramics (PDCs) route and the Spark Plasma Sintering (SPS) process is proposed, leading to well-crystallized and pure layered h-BN crystals, prone to be exfoliated into large BNNSs. Here we focus more specifically on the influence of two key-parameters of the process to be optimized: the $\mathrm{Li}_{3} \mathrm{~N}$ concentration $(0-10 \mathrm{wt} . \%)$ and the sintering temperature $\left(1200-1950^{\circ} \mathrm{C}\right)$. The presence of $\mathrm{Li}_{3} \mathrm{~N}$, added as crystal promoter in the preceramic powder, significantly improves the crystallinity level of the product, as evidenced by XRD, SEM and Raman spectrometry. Besides, sintering temperature strongly modifies the size of resulting h-BN flakes. Then, influence of sintering temperature on both purity and crystallinity is studied using a performing tool, the cathodoluminescence. h-BN flakes larger than $200 \mu \mathrm{m}^{2}$ (average flake area) are obtained. Few-layered BNNSs are successfully isolated, through exfoliation process.
\end{abstract}

Keywords: Two-dimensional Materials, Graphene Substrates, h-BN, Spark Plasma Sintering, BNNSs, Polymer Derived Ceramics 


\section{Introduction}

Since 2004, when scientists, for the first time, were able to isolate graphene nanosheets from graphite,[1] synthesis and properties of graphene have become keystones of worldwide research. Meanwhile, prompted by the rising star of graphene, the related 2D nanomaterials became a hot issue in the literature, such as hexagonal BN (h-BN), transition metal oxides (TMOs) and transition metal dichalcogenides (TMDs).[2-5] Among them, the hexagonal boron nitride nanosheets (BNNSs) consist of thin atomic layers made of $\mathrm{B}$ and $\mathrm{N}$ atoms covalently bounded in plane. BNNSs have shown to be an excellent gate dielectric support for graphene and other twodimensional materials owing to their atomically smooth surface, high thermal conductivity and stability, combined with high mechanical strength. Compared with conventional $\mathrm{SiO}_{2} \mathrm{Substrate}_{\text {, }}$ lattice matching and absence of dangling bonds make BNNSs and graphene excellent pairing materials, and give incentive to develop various van der Waals hetero-structures.[6, 7] However, it has to be pointed out that such applications cannot be put into use without high purity large BNNSs.

To obtain large and pure BNNSs, Chemical Vapor Deposition (CVD) and exfoliation of BN crystals are generally considered. In the first method, different precursors could be used for the CVD synthesis. Among them, borazine is the main option, because it is isostructural to benzene.[8] The growth of BNNSs has been carried out primarily on transition metal substrates, such as $\mathrm{Cu}$, $\mathrm{Ni}, \mathrm{Fe}$ and so on.[9-12] This is based on their catalytic effect at high temperatures. In recent years, mono- and few-layered BNNSs with domains size up to centimeter-scale have been reported.[1317] It should be mentioned that, CVD and CVD-derivative synthesis of BNNSs are strongly related to the morphology of substrates and purity of chemicals. Domain size, morphology and layer number are still the challenges for the applications of CVD BNNSs.[8, 18-20]

Another approach is a common method for obtaining 2D materials, which takes advantage of the weak inter-planar sheets forces. Obtaining BNNSs by exfoliation is greatly relevant to the quality of h-BN crystal source. Nevertheless, commercial sources are unreliable because of their highlevel of defects and small crystal size, which are from $1 \mu \mathrm{m}$ to $10 \mu \mathrm{m}$. The group of Watanabe and Taniguchi at the National Institute for Materials Science (NIMS) is able to prepare high quality 
crystals using their own High Pressure High Temperature (HPHT) method. This method needs to be conducted through severe conditions and long synthesis time.[21]

In order to achieve high quality large BNNSs, our group has already proposed preliminary results on a novel synthesis way by the Polymer Derived Ceramics (PDCs) route involving polyborazylene (PBN) as precursor, combined with the Spark Plasma Sintering (SPS).[22] Compared with samples from Saint-Gobain, HQ and CVD thin films, our h-BN sample exhibited better FWHM of Raman high frequency peak ( $E_{2 g}$ mode).[23, 24] Here we present further investigations on how to optimize the process, considering the influence, on h-BN growth, of both sintering temperature and crystallization promoter. The PDCs route consists in the synthesis of very pure preceramic polymer from tailored molecular precursor in a non-oxide system. This intermediate compound (liquid, soluble or fusible) can be then easily shaped into different forms. Based on its relatively high ceramic yield, high purity and convenient $\mathrm{B} / \mathrm{N}$ atomic ratio, $\mathrm{PBN}$ is ideal to get further stoichiometric h-BN through an appropriate thermal post-treatment. Due to its rapid heating, the highly energetic SPS process is considered as an efficient method for the densification of advanced powder-based materials. Compared with conventional sintering techniques, it is indeed possible to achieve near-theoretical density at lower sintering temperature, lower pressure and shorter dwelling duration. To date, densification of polymer-derived $\mathrm{SiC}$, SiO-C, Si-C-N and Si-B-C-N ceramics by SPS has been seldom investigated.[25-28]

\section{Experimental method}

The preparation of PBN was conducted following a procedure described elsewhere.[29] Role of crystallization promoter was studied by dispersing lithium nitride $\left(\mathrm{Li}_{3} \mathrm{~N}, 99.5 \%\right.$, Aldrich) micro powders into pure homemade liquid polyborazylene (PBN).[30] Based on previous study,[31] a series of dispersive solutions with different concentrations of $\mathrm{Li}_{3} \mathrm{~N}(0 \%, 5 \%$ and 10 wt. $\%)$ were prepared. These dispersions were slowly heated into a glove box filled with argon from room temperature to $200^{\circ} \mathrm{C}$, and hold at this temperature for $1 \mathrm{~h}$ leading to a solid-state polymer avoiding further oligomer evaporation. The resulting white powder was then placed into an alumina crucible to be heated under $\mathrm{N}_{2}$ at $650{ }^{\circ} \mathrm{C}\left(3^{\circ} \mathrm{C} \cdot \mathrm{min}^{-1}\right)$, then kept at this final temperature for $1 \mathrm{~h}$. 
For SPS experiments, a protective anti-sticking papyex ${ }^{\circledR}$ sheet was firstly covered on the inner surface of the $10 \mathrm{~mm}$ diameter SPS graphite die. The graphite die was filled with the preceramic powder in a glove box (300-400 mg) and then transferred into the SPS chamber (HP D 25, FCT System) in-between the two punches, then compressed at room temperature under uniaxial load until $90 \mathrm{MPa}$ (see Figure 1). SPS heating was then conducted at different temperatures (1200 $\left.1950{ }^{\circ} \mathrm{C}\right)$ with the same heating rate $\left(100{ }^{\circ} \mathrm{C} \cdot \mathrm{min}^{-1}\right)$ maintaining the $90 \mathrm{MPa}$ load throughout the 1-hour sintering. This process time was defined from preliminary experiments based on XRD results, which have revealed that too short times (10 minutes) gave rise to a poorly crystallized product, while higher durations (6 hours) did not improve crystallinity. During the sintering, SPS chamber vacuum was $0.1-0.4 \mathrm{hPa}$. The heating process was held at this maximum temperature to complete the $\mathrm{BN}$ ceramization reaction and the densification process.

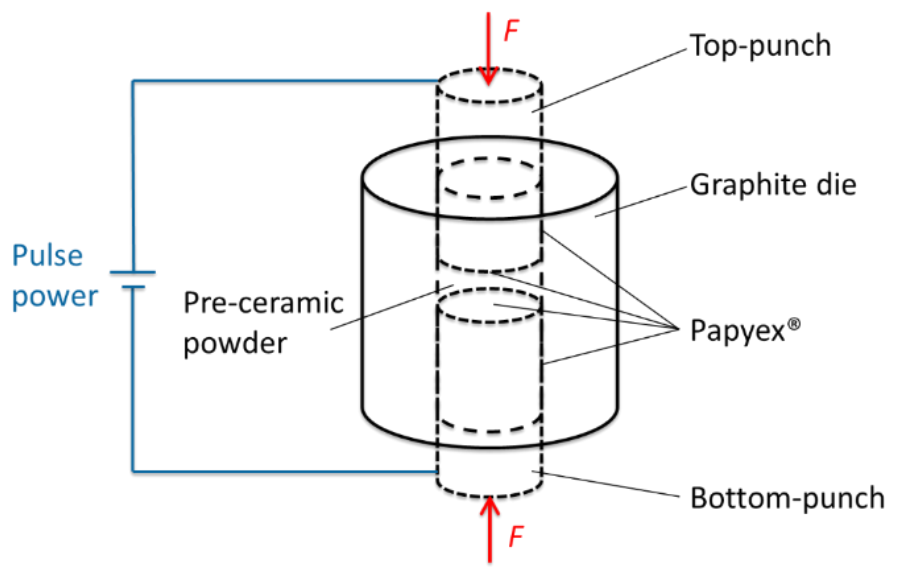

Figure 1. Schematic diagram of SPS experimental set-up

As-sintered h-BN pellets and exfoliated BNNSs were characterized from both chemical and microstructural viewpoints. Scanning Electron Microscopy (SEM) observations were carried out at very low accelerating voltage $(800 \mathrm{~V})$ using a SUPRA Zeiss 55VP microscope. A statistic size distribution was achieved by processing SEM images through the ImageJ software, analyzing average area of 100 well-oriented h-BN flakes for each sintered pellet. The Raman scattering spectra were recorded in backscattering geometry using a high resolution Raman spectrometer setup (Labram HR800 from HORIBA Jobin-Yvon), using an excitation wavelength of $514.5 \mathrm{~nm}$ 
with a x100 objective. Mean and standard deviation were calculated over typically 100 spectra. XRay Diffraction (XRD) patterns were recorded with a Bruker D8 diffractometer, using the CuK radiation source. Cathodoluminescence (CL) was performed on $300 \mu \mathrm{m}$ thick cross sections of each sample, prepared using a wire saw. At the end, a $5 \mathrm{~nm}$ gold film was deposited on the insulating hBN samples to avoid electrical charging during electron-beam irradiation. CL experiments rely on a JEOL7001F field-emission-gun scanning electron microscope (SEM). A parabolic mirror collects the light, which is further analyzed with a TRIAX550 Horiba Jobin-Yvon spectrometer equipped with a 600 grooves/mm grating. All samples were mounted together on a GATAN cold-finger cryostat cooled with liquid helium. Spectra are acquired with a $3 \mathrm{keV}$ and 1 $\mathrm{nA}$ electron beam at a $10 \mathrm{~K}$ cryostat temperature. The UV luminescence efficiency from exciton lines around $215 \mathrm{~nm}$ (S-series) was extracted following a procedure described elsewhere.[32] To assess a relevant analysis of the polycrystalline samples, CL spectra were acquired by exciting a large surface $\left(1750 \mu \mathrm{m}^{2}\right)$ providing representative CL spectra, averaged over many crystals. BNNSs are prepared through a chemical procedure (in ethanol, 2 min ultrasonication, Hielscher UP400S). A droplet of the solution was simply deposited onto a 300 mesh carbon copper grid in order to perform Transmission Electron Microscopy (TEM) observations and Selective Area Electron Diffraction (SAED) using a JEOL 2100F microscope operating at $200 \mathrm{kV}$.

\section{Results and discussion}

\subsection{Influence of crystallization promoter}

The alkaline and alkaline earth metals such as lithium nitrides were first used as catalysts for synthesis of c-BN by Wendorf in 1960s. [33] Since then, $\mathrm{Li}_{3} \mathrm{~N}$ has become a traditional promoter for transforming h-BN to c-BN.[34, 35] Besides c-BN, $\mathrm{Li}_{3} \mathrm{~N}$ is also reported for a better h-BN crystallization level.[6, 36]

Owing to the further addition of $\mathrm{Li}_{3} \mathrm{~N}$ to the reactant powder, the final amount of h-BN got is affected. Product yield values (mass obtained over expected mass for the whole conversion of PBN to h-BN) calculated for the three samples sintered at $1800^{\circ} \mathrm{C}$, based on weight measurements, appear Table 1. During the ceramization process of PBN giving rise to $h-B N, \mathrm{a}_{2}$ release occurs, explaining the reason why the product yield of the $\mathrm{Li}_{3} \mathrm{~N}$-free sample is different to $100 \%$ (93\%). 
With the increase of $\mathrm{Li}_{3} \mathrm{~N}$ addition in the pre-ceramic powder, the yield decreases drastically down to $61 \%$ for a $\mathrm{Li}_{3} \mathrm{~N}$ content of $10 \%$. In addition to the expected $\mathrm{H}_{2}$ release, more $\mathrm{Li}_{3} \mathrm{~N}$ added leads to a higher amount of intermediates, which are lost during the sintering step. Weight loss can be attributed to the reaction between $\mathrm{PBN}$ and $\mathrm{Li}_{3} \mathrm{~N}$, reforming the intermediate compound, $\mathrm{Li}_{3} \mathrm{BN}_{2}$, to be eliminated.[30]

Table 1: Product yield of SPS pellet compared with pre-ceramic powder

\begin{tabular}{cccc}
\hline $\begin{array}{c}\mathrm{Li}_{3} \mathrm{~N} \text { Concentration } \\
\text { wt } \%\end{array}$ & 0 & 5 & 10 \\
\hline $\begin{array}{c}\text { Product Yield } \\
\%\end{array}$ & 93 & 80 & 61 \\
\hline
\end{tabular}



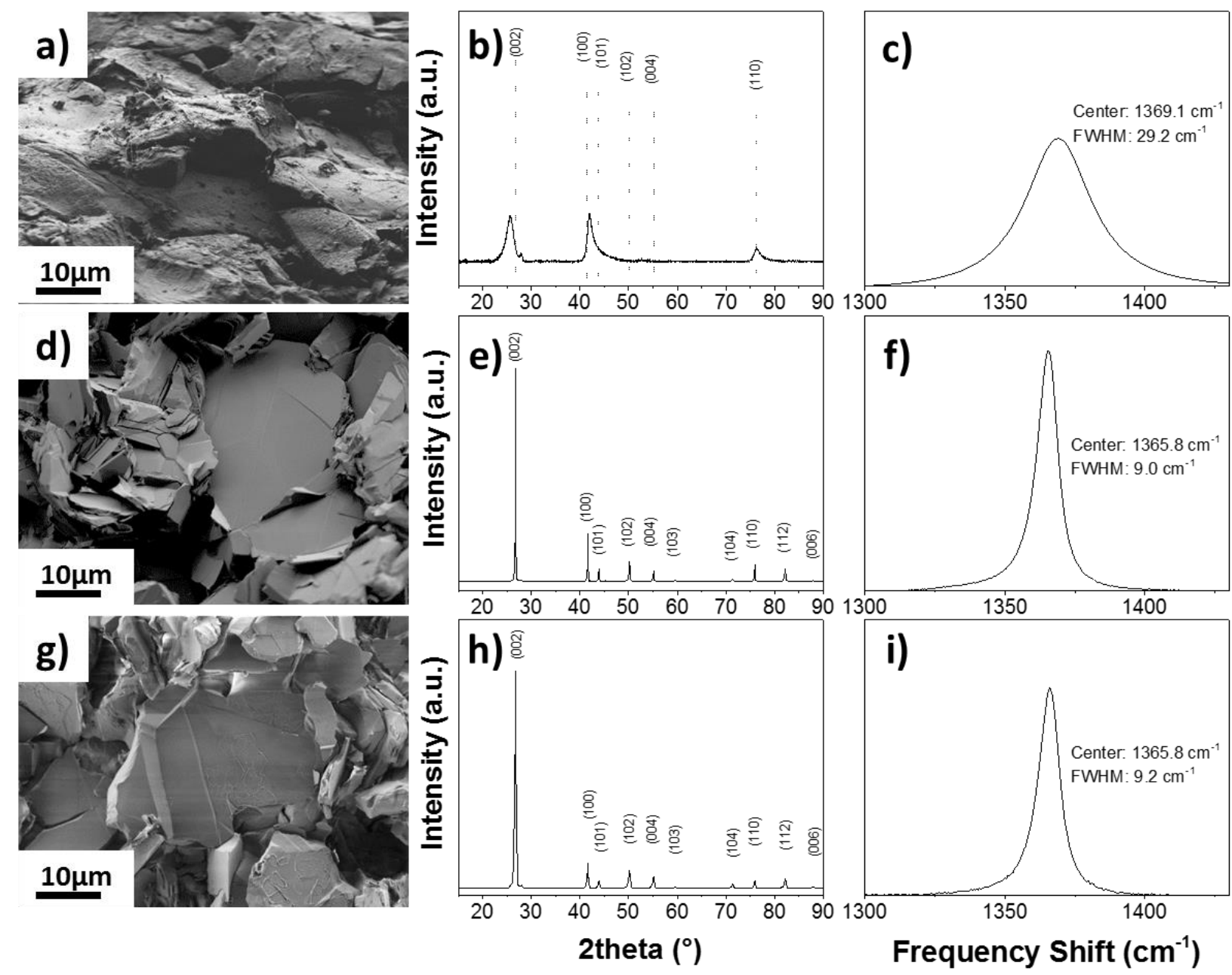

Figure 2. Microstructural and chemical characterization of the product obtained for a $1800^{\circ} \mathrm{C}$ sintering temperature, without $\mathrm{Li}_{3} \mathrm{~N}$ (a-c), with 5 wt.\% (d-f) and 10 wt.\% $\mathrm{Li}_{3} \mathrm{~N}(\mathrm{~g}-\mathrm{i}): \mathrm{a}, \mathrm{d}, \mathrm{g}$ : SEM observation; b,e,h: XRD patterns; c,f,i: Raman spectra.

Figure 2 presents the microstructural and chemical characteristics of the samples prepared with different amounts of $\mathrm{Li}_{3} \mathrm{~N}$ added to the pre-ceramic powder and for a sintering step conducted at $1800^{\circ} \mathrm{C}$. Without $\mathrm{Li}_{3} \mathrm{~N}$, an "amorphous-like" disordered structure is observed, composed of undefined blocks (Fig. 2a). Interpretation of the XRD patterns confirms the very poor structural organization of the agglomerated product (Fig. 2b). Indeed, even if a reaction occurred during the 
SPS process, the resulting boron nitride is poorly crystallized with only three broad diffused peaks. It is worth mentioning that the (001) peak, particularly well defined for h-BN and characteristic of its 2-dimensional nature, does not evidence in this case any preferential texture. Such a poor quality of boron nitride, resulting from a partially transformation of pre-ceramic powder, is also corroborated by the Raman investigation, showing the $E_{2 \mathrm{~g}}$ mode peak centered at $1369.1 \mathrm{~cm}^{-1}$ and a Full Width at Half Maximum (FWHM) value of $29.2 \mathrm{~cm}^{-1}$. On the opposite, when $5 \mathrm{wt} \%$ or 10 wt.\% $\mathrm{Li}_{3} \mathrm{~N}$ is mixed with PBN (Figure $2 \mathrm{~d}, 2 \mathrm{~g}$ respectively), well-defined and sharp single peaks are observed. This proves the high efficiency of lithium nitride as crystal promoter, leading to well crystallized stacked BN. Considering the Raman spectra, the quality of h-BN regarding its structural and chemical characteristic is comparable. However, the XRD patterns show a bigger crystal size at $5 \mathrm{wt} . \%$ than at $10 \mathrm{wt}$ \% as proved by the lower FWHM of the (002) diffraction peak $\left(0.12^{\circ}\right.$ for $5 \mathrm{wt} . \%$ and $0.44^{\circ}$ for $\left.10 \mathrm{wt} . \%\right)$. It is worth mentioning that this value is significantly lower than that presented in other study[37]. Moreover, the effect of $\mathrm{Li}_{3} \mathrm{~N}$ on the flakes growth is obvious, the flakes mean dimension being modified between the two $\mathrm{Li}_{3} \mathrm{~N}$ investigated contents (276 $\mu \mathrm{m}^{2}$ for $5 \mathrm{wt} . \%$ to be compared with $215 \mu \mathrm{m}^{2}$ for $10 \mathrm{wt} . \%$ ).

As a partial conclusion, the $\mathrm{Li}_{3} \mathrm{~N}$ addition is necessary for obtaining well-crystalized h-BN flakes. No significant difference is noted between $5 \%$ and $10 \mathrm{wt} \%$, so that we have selected the lowest $\mathrm{Li}_{3} \mathrm{~N}$ amount, maintaining the highest ceramic yield, for the sintering temperature study.

\subsection{Influence of sintering temperature}

To determine the role of the SPS temperature and according to the previous results, all samples of this part were prepared with a powder containing $5 \mathrm{wt} . \% \mathrm{Li}_{3} \mathrm{~N}$. The as-sintered samples are $10 \mathrm{~mm}-$ diameter pellets, which are characterized adopting a multiscale approach, involving SEM, XRD, Raman and cathodoluminescence (CL) investigations.

First, a microscopic characterization was conducted. Figure 3 present a series of representative SEM images of raw materials sintered at different SPS temperatures. Whatever the temperature, pellets show similar feature composed of defined $\mathrm{BN}$ domains staked into flakes and growing in different orientations. 


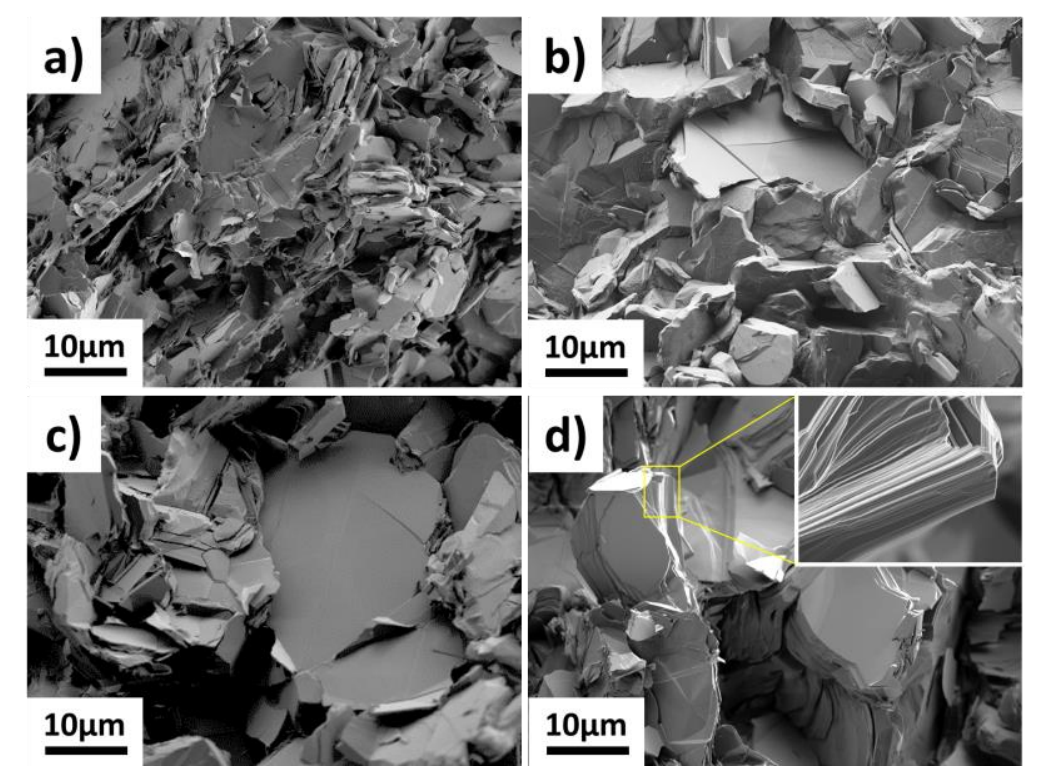

Figure 3: Cross-section SEM images of BN pellets

a) $1200^{\circ} \mathrm{C}$; b) $1500{ }^{\circ} \mathrm{C}$; c) $1800^{\circ} \mathrm{C}$; d) $1950{ }^{\circ} \mathrm{C}$

(laminar structure shown in the insert image)

However, at the lowest temperature of $1200^{\circ} \mathrm{C}$, it appears that the flakes show smaller lateral size compared to ones synthetized above. The lowest temperature gives rise to small micrometer-scale flakes (Fig. 3a). For higher sintering temperatures, flakes are significantly larger. It can be noticed that at higher magnification, BN flakes clearly show the characteristic laminar structure, prone to a further exfoliation (e.g. insert in Fig. 3d). Their dimension is difficult to determine quantitatively, however a distribution of the flakes size was estimated through a statistical approach, considering 100 flakes. The distribution graphs are presented in Figure 4. The flakes size increases with the temperature to reach a maximum area of $276 \mu \mathrm{m}^{2}$ at $1800^{\circ} \mathrm{C}$, and more than half of flakes population is characterized by an area exceeding $250 \mu \mathrm{m}^{2}$. At $1950^{\circ} \mathrm{C}$ (the highest temperature allowed by the graphite die in our SPS machine at 90MPa), flakes dimension decreases a little to $245 \mu^{2}$. One explanation could be that at higher sintering temperature, the nucleation rate increases faster than the crystal growth rate, thus decreasing the flakes size. However, sintering temperatures between 1500 and $1800^{\circ} \mathrm{C}$ seem to be a good compromise between these two opposite behaviors (number of nucleation sites versus growth kinetic). 


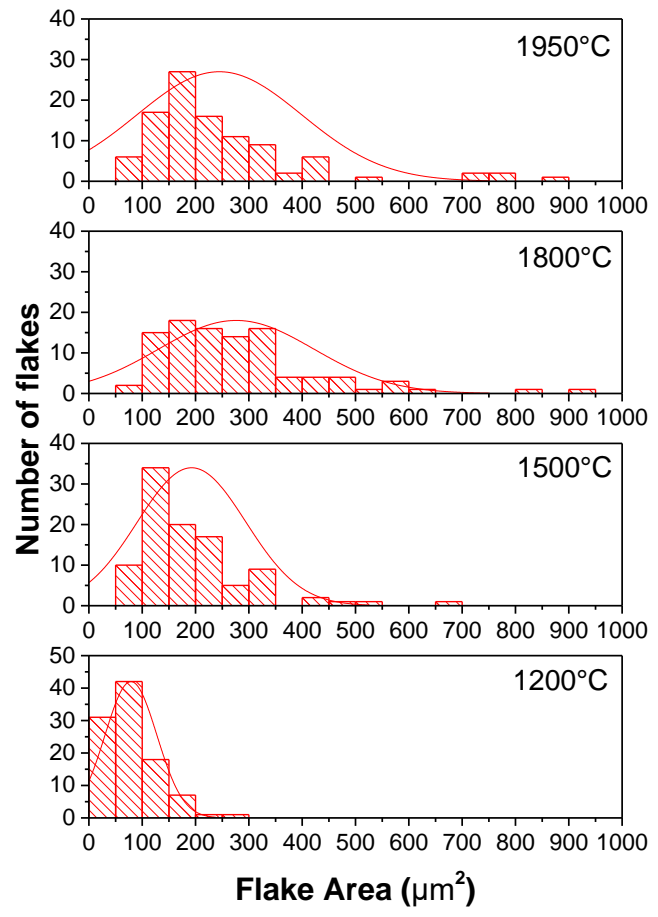

Figure 4: Flakes size distribution of sintered pellets

The crystalline organization of the flakes was confirmed by a deeper investigation through XRD. 

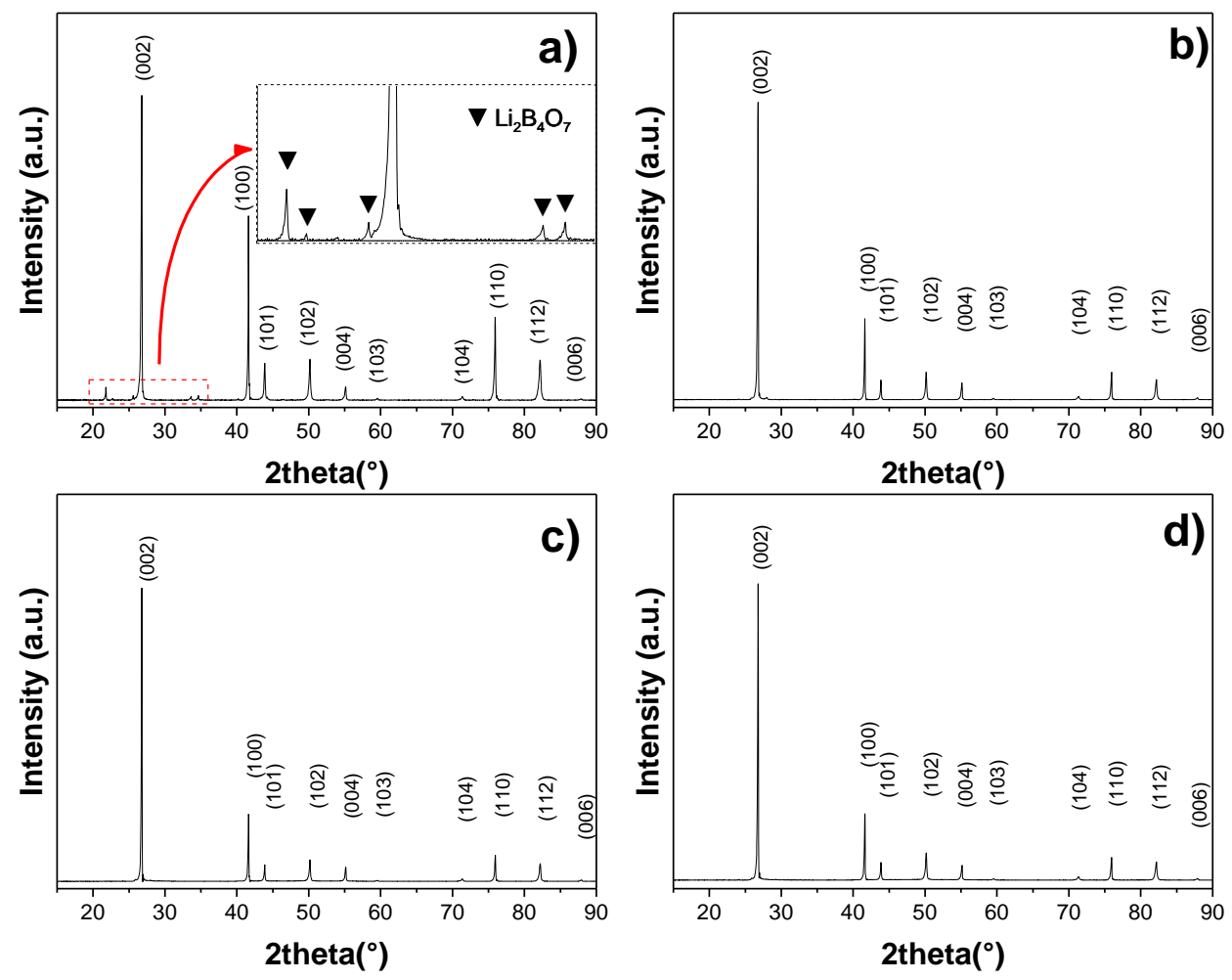

Figure 5: XRD patterns of sintered pellets

a) $1200^{\circ} \mathrm{C}$; b) $1500{ }^{\circ} \mathrm{C}$; c) $1800^{\circ} \mathrm{C}$; d) $1950{ }^{\circ} \mathrm{C}$

Figure 5 presents XRD patterns of pellets sintered at $1200^{\circ} \mathrm{C}, 1500^{\circ} \mathrm{C}, 1800^{\circ} \mathrm{C}$ and $1950^{\circ} \mathrm{C}$. All patterns still exhibit thin and well-separated peaks, indicating a good crystalline structure. Besides, it is worth noting the major contribution of the (002) peak, which highlights the strong 2dimensional character of the product. Except for the sample sintered at $1200{ }^{\circ} \mathrm{C}$, all peaks are assigned to h-BN crystallographic planes in accordance with the 00-034-0421 JCPDS file. For the sample sintered at the lowest temperature, in addition to the representative h-BN peaks, $\mathrm{Li}_{2} \mathrm{~B}_{4} \mathrm{O}_{7}$ (JCPDS 00-018-0717) very slight peaks also appeared within the $\left[20,35^{\circ}\right] 2 \theta$ angular region (Fig. 5a). This compound may result from the reaction between $\mathrm{PBN}, \mathrm{Li}_{3} \mathrm{~N}$ and little amount of $\mathrm{O}_{2}$ impurity, which could have been adsorbed on the surface of the reactor and the pellet prior to the chamber vacuum. $\mathrm{Li}_{2} \mathrm{~B}_{4} \mathrm{O}_{7}$ starts to melt at $917^{\circ} \mathrm{C},[38]$ but traces of this oxide are still recorded at $1200^{\circ} \mathrm{C}$. This compound is nevertheless totally degraded at $1500^{\circ} \mathrm{C}$, since its corresponding XRD pattern does not show any traces of Li-based compounds anymore (Fig. 5b). This means that, 
although little $\mathrm{O}_{2}$ may react with the precursor during the sintering process, this undesirable oxide is likely to be removed out of the pellet together with the $\mathrm{H}_{2}$ flow, as expected during the ceramization process.[29] From these first results, one can expect that sintering process should be performed above $1500^{\circ} \mathrm{C}$ to avoid any lithium-based contamination.
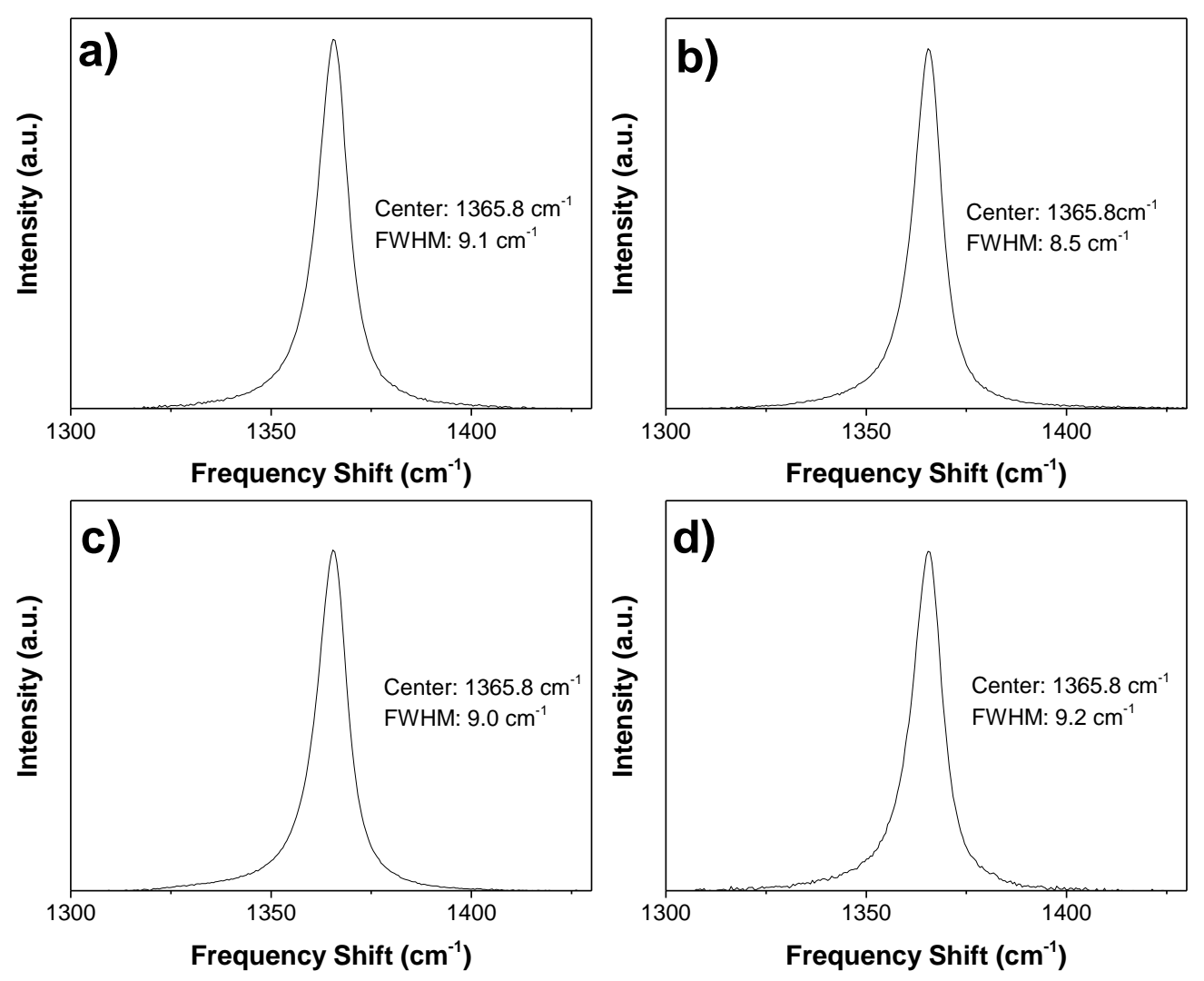

Figure 6: Raman spectra of sintered pellets

a) $1200^{\circ} \mathrm{C}$; b) $1500{ }^{\circ} \mathrm{C}$; c) $1800^{\circ} \mathrm{C}$; d) $1950{ }^{\circ} \mathrm{C}$

h-BN crystal quality has been characterized by Raman spectroscopy. All spectra, presented in Figure 6, show narrow well-defined single peaks, centered on the expected $1365.8 \mathrm{~cm}^{-1}$ frequency shift. Homogeneity was investigated by performing horizontal and vertical line scans on pellet cross-sections. Mean and standard deviation were calculated over typically 100 spectra giving rise to relevant values. 


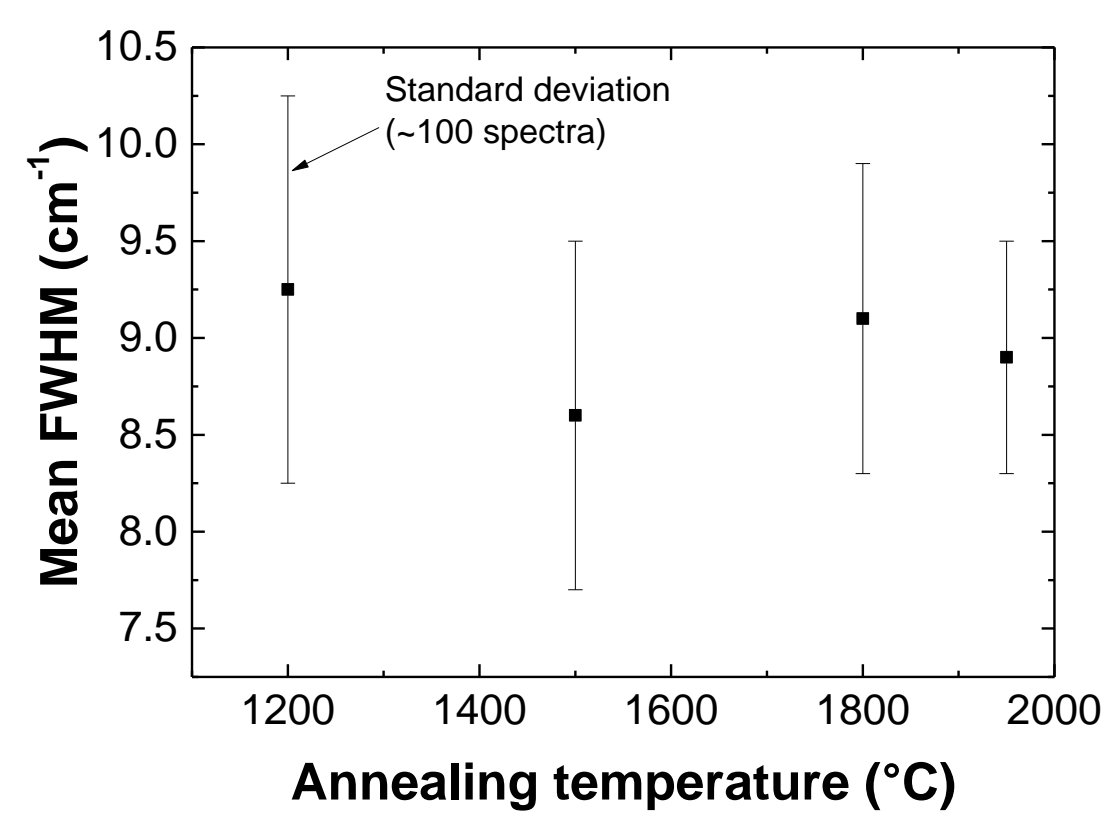

Figure 7: Mean values and standard deviations of the $\mathrm{E}_{2 \mathrm{~g}}$ peak FWHM as a function of the sintering temperature

Figure 7 shows a graph presenting the mean value and the standard deviation of the FWHM of the $\mathrm{E}_{2 \mathrm{~g}}$ peak as a function of the sintering temperature. It appears that the standard deviations are typically around 0.6 to $1 \mathrm{~cm}^{-1}$ due to variations from grain to grain inside one sample. The mean FWHM values are centered on $9 \mathrm{~cm}^{-1}$ with the lowest value at $8.5 \mathrm{~cm}^{-1}$ for the $1500^{\circ} \mathrm{C}$ sample. It can be noted that under the same operating conditions, our best value is close to the one of reference samples from NIMS and others.[24, 37, 39]

Recently, it has been demonstrated that $\mathrm{CL}$ is a much richer characterization method for h-BN crystals than the Raman single peak $\left(1366 \mathrm{~cm}^{-1}\right)$ and FWHM analysis, usually performed in nonresonant conditions.[24] This characterization technique is very sensitive to deep structural and chemical defects, and in this sense is complementary to XRD and Raman measurements. In this context, average CL spectra were recorded on pellet cross-sections exciting a sufficiently large area to analyze many grains. In these conditions, no significant variations are found inside the pellet volume, and the representative CL spectra are presented and analyzed in Fig. 8. 

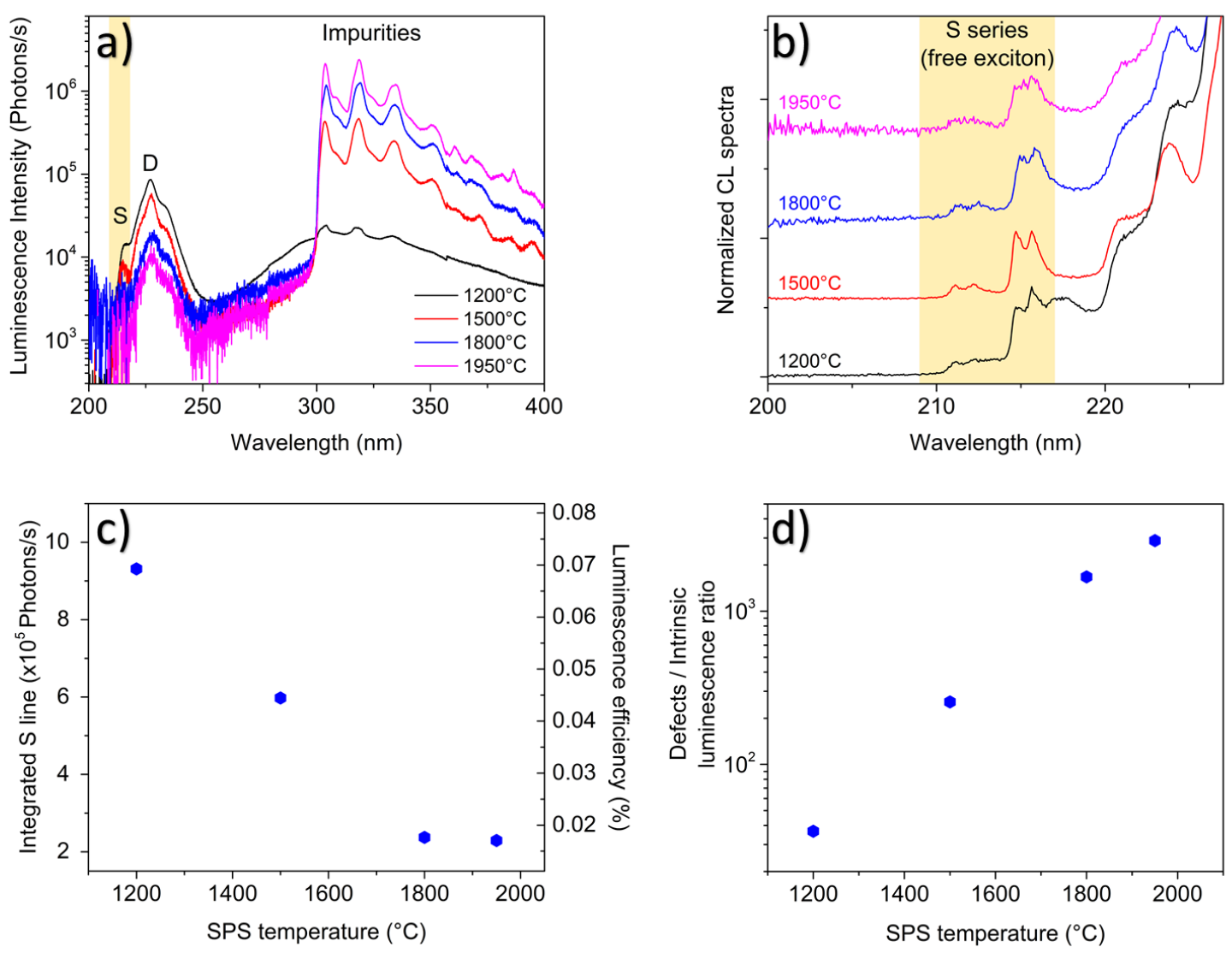

Figure 8: CL results showing a) low spectral resolution, b) high spectral resolution, c) UV luminescence efficiency versus SPS temperature, d) CL of impurities compared to free excitons versus SPS temperature

Fig.8(a) first gives a spectrum overview for CL intensity comparisons. Except with the low temperature annealing at $1200^{\circ} \mathrm{C}$, luminescence from impurities with a zero-phonon-line at 302 $\mathrm{nm}$ predominates. Such luminescence from middle gap energy states is related to point defect centers containing carbon or oxygen as already shown by other authors.[40-42] The so-called D series attributed to excitons trapped at structural defects is also observed with a maximum at 227 $\mathrm{nm}$. Finally, the intrinsic luminescence of the h-BN free excitons is observed around $215 \mathrm{~nm}$, as better shown at high resolution on Fig. 8(b) revealing phonon-assisted lines of indirect excitons (S series). 
As in conventional semiconductors, the efficiency of intrinsic light emissions provides an efficient way to indicate the overall material quality, accounting for both purity and crystallinity. Fig.8(c) shows that the measured luminescence efficiency (LE) decreases strongly when using high temperatures for the sintering process. Such an interpretation is supported by the ratio between the impurity-related and the intrinsic luminescence drawn in Fig. 8(d), which increases by 2 orders of magnitude from $1200^{\circ} \mathrm{C}$ to $1950^{\circ} \mathrm{C}$ annealing temperatures. This is most probably due to a nonintentional thermally activated diffusion of impurities in h-BN pellet, such as carbon and oxygen. Presence of these elements at a level of the atomic percent has indeed already been reported in the literature [22], and has to be associated with graphite parts in the SPS reactor and with the primary vacuum involved during the sintering operation.

As a summary, the highest SPS temperatures promote larger flakes with better structural quality as shown by Raman spectroscopy. However, CL shows that high temperatures also induce a contamination of $\mathrm{h}-\mathrm{BN}$ crystals by impurities. This work opens the way to an optimization of the SPS process, matter of compromise between crystallinity and flake sizes.

\subsection{Towards h-BN nano-sheets}

The main goal of the study was to optimize the experimental conditions in order to give rise to well-crystallized and large h-BN flakes, prone to be exfoliated into high quality BNNSs. Actually, a SPS sintering of a pre-ceramic powder including $5 \mathrm{wt} \% \mathrm{Li}_{3} \mathrm{~N}$ performed between 1500 and $1800^{\circ} \mathrm{C}$ allows getting well-crystallized, low impurity content h-BN flakes larger than $200 \mu \mathrm{m}^{2}$ (average area) with a good yield (80\%). These flakes were then dispersed in ethanol and chemically exfoliated for TEM observations (Figure 9). The micrograph acquired at low magnification shows a representative several microns sheet (Fig. 9a). Its size is by far lower than that of original flakes, which may be explained by their damage under external force generated by sonication during the exfoliation process. Nevertheless, the above-mentioned high quality of h-BN is also shown at atomic scale. We indeed observe few-layered (less than 10) uniform BNNSs, with an hexagonal structure clearly confirmed by SAED, which shows six bright spots hexagonally distributed 
(Figure 9b and insert). This corresponding SAED pattern is interpreted as the AA' atomic stacking in the c-direction.
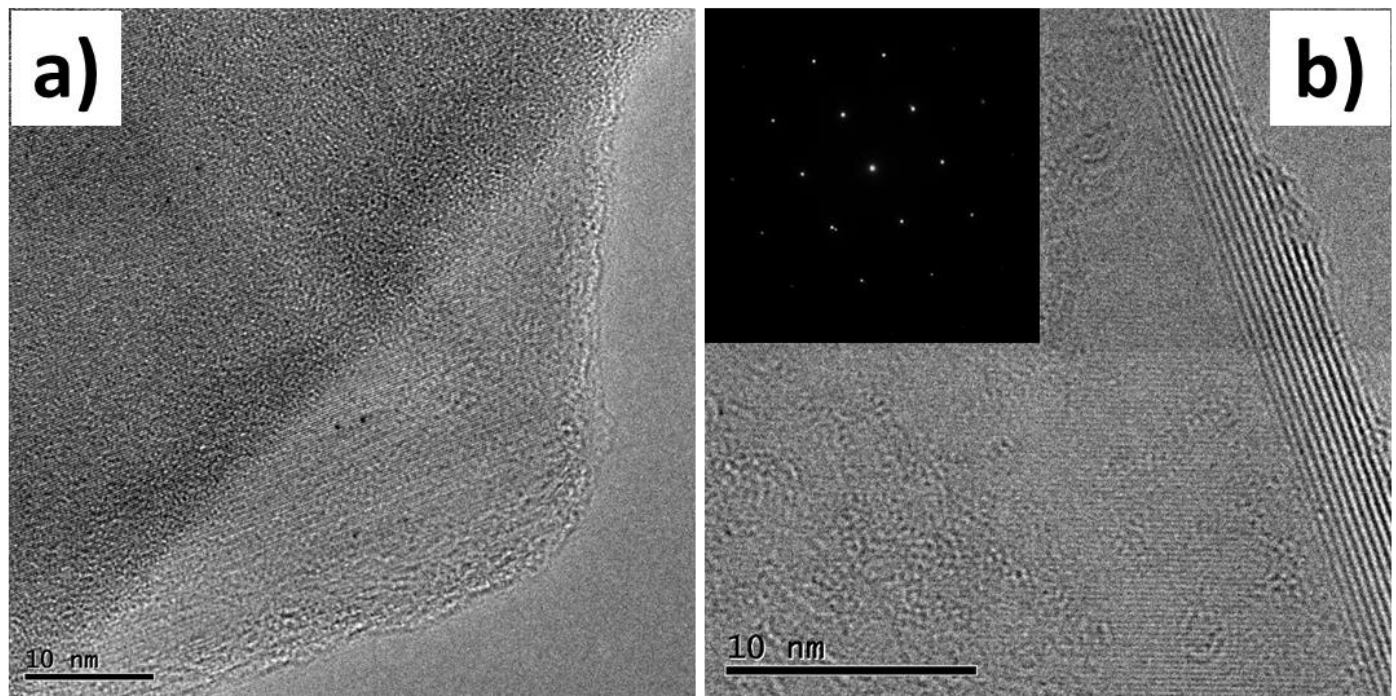

Figure 9. TEM images of exfoliated BNNSs from the $1800{ }^{\circ} \mathrm{C} 5 \mathrm{wt} . \% \mathrm{Li}_{3} \mathrm{~N}$ sintered pellet

\section{Conclusion}

This study aimed to better understand and optimize an innovative laboratory-scale way to produce hexagonal BNNSs through a combination of PDCs route and SPS process. The two key-parameters, temperature and $\mathrm{Li}_{3} \mathrm{~N}$ contents, were investigated.

Without $\mathrm{Li}_{3} \mathrm{~N}$, a single-blocked poorly crystallized material is obtained. For a 5 or $10 \mathrm{wt} . \% \mathrm{Li}_{3} \mathrm{~N}$ addition to the PBN powder, structural and chemical characteristics are excellent. However, the further $\mathrm{Li}_{3} \mathrm{~N}$ reactant also affects the product yield owing to the elimination of intermediate compounds. The $5 \mathrm{wt} . \%$ addition of $\mathrm{Li}_{3} \mathrm{~N}$ presents thus the best compromise between the amount of h-BN got, and its crystallinity.

It has also been established that at too low temperature $\left(1200^{\circ} \mathrm{C}\right)$, some intermediate reaction products still remain in the resulting pellet, like $\mathrm{Li}_{2} \mathrm{~B}_{4} \mathrm{O}_{7}$, showing that $\mathrm{Li}_{3} \mathrm{~N}$ may act as an oxygen gas getter. From $1500^{\circ} \mathrm{C}$, the reaction seems completed and the by-products eliminated, giving rise to high-quality well-crystallized h-BN staked flakes with average flake area more than $200 \mu \mathrm{m}^{2}$. 
The best experimental conditions (sintering from 1500 to $1800^{\circ} \mathrm{C}$ with $5 \mathrm{wt} . \% \mathrm{Li}_{3} \mathrm{~N}$ enriched preceramic powder) lead to flakes around $250 \mu \mathrm{m}^{2}$ on average. XRD and Raman spectra prove good crystal quality even if CL detects some traces of contamination, when increasing the sintering temperature. We are now optimizing the exfoliation step in order to deliver larger sheets, whose size will be comparable to that of original flakes.

\section{Acknowledgement}

This work was financially supported by ANR through the project 14-CE08-0018 «GoBN», European Union Horizon 2020 Program under the Graphene Flagship (grant number 604391), and Chinese Scholarship Council (CSC). Authors also thank the CT $\mu$ (Centre Technologique des Microstructures) of the Université Claude Bernard Lyon 1 and the CLYM (Consortium Lyon Saint-Etienne de Microscopie) for access to microscopies.

\section{Conflicts of interest}

The authors declare no conflict of interest.

\section{References}

1. Novoselov K.S., Geim A.K., Morozov S.V., Jiang D., Zhang Y., Dubonos S.V., Grigorieva I.V. and Firsov A.A. Electric Field Effect in Atomically Thin Carbon Films. Science, 2004. 306(5696): 666-669.

2. $\quad$ Novoselov K.S., Jiang D., Schedin F., Booth T.J., Khotkevich V.V., Morozov S.V. and Geim A.K. Two-dimensional atomic crystals. PNAS, 2005. 102(30): 10451-10453.

3. Takada K., Sakurai H., Takayama-Muromachi E., Izumi F., Dilanian R.A. and Sasaki T. Superconductivity in two-dimensional CoO2 layers. Nature, 2003. 422(6927): 53-55.

4. Golberg D., Bando Y., Huang Y., Terao T., Mitome M., Tang C.C. and Zhi C.Y. Boron Nitride Nanotubes and Nanosheets. Acs Nano, 2010. 4(6): 2979-2993.

5. Wang Q.H., Kalantar-Zadeh K., Kis A., Coleman J.N. and Strano M.S. Electronics and optoelectronics of two-dimensional transition metal dichalcogenides. Nature Nanotechnology, 2012. 7(11): 699-712. 
6. Lee G.H., Yu Y.J., Lee C., Dean C., Shepard K.L., Kim P. and Hone J. Electron tunneling through atomically flat and ultrathin hexagonal boron nitride. Applied Physics Letters, 2011. 99(24): 3.

7. Woessner A., et al. Highly confined low-loss plasmons in graphene-boron nitride heterostructures. Nature Materials, 2015. 14(4): 421-425.

8. Khan M.H., Liu H.K., Sun X., Yamauchi Y., Bando Y., Golberg D. and Huang Z. Fewatomic-layered hexagonal boron nitride: CVD growth, characterization, and applications. Materials Today, 2017. 20(10): 611-628.

9. Ismach A., Chou H., Ferrer D.A., Wu Y., McDonnell S., Floresca H.C., Covacevich A., Pope C., Piner R. and Kim M.J. Toward the controlled synthesis of hexagonal boron nitride films. ACS nano, 2012. 6(7): 6378-6385.

10. Kim K.K., Hsu A., Jia X., Kim S.M., Shi Y., Hofmann M., Nezich D., Rodriguez-Nieva J.F., Dresselhaus M. and Palacios T. Synthesis of monolayer hexagonal boron nitride on $\mathrm{Cu}$ foil using chemical vapor deposition. Nano letters, 2012. 12(1): 161-166.

11. Park S., Lee J., Kim H.S., Park J.-B., Lee K.H., Han S.A., Hwang S., Kim S.-W. and Shin H.-J. Formation of Hexagonal Boron Nitride by Metal Atomic Vacancy-Assisted BN Molecular Diffusion. ACS nano, 2015. 9(1): 633-638.

12. Caneva S., et al. Controlling Catalyst Bulk Reservoir Effects for Monolayer Hexagonal Boron Nitride CVD. Nano Letters, 2016. 16(2): 1250-61.

13. Park J.-H., Park J.C., Yun S.J., Kim H., Luong D.H., Kim S.M., Choi S.H., Yang W., Kong J. and Kim K.K. Large-Area Monolayer Hexagonal Boron Nitride on Pt Foil. ACS nano, 2014. 8(8): 8520-8528.

14. Oh H., Jo J., Tchoe Y., Yoon H., Hwi Lee H., Kim S.-S., Kim M., Sohn B.-H. and Yi G.C. Centimeter-sized epitaxial h-BN films. NPG Asia Materials, 2016. 8(11): e330-e330.

15. Corso M., Auwarter W., Muntwiler M., Tamai A., Greber T. and Osterwalder J. Boron nitride nanomesh. Science, 2004. 303(5655): 217-220.

16. Lu G., Wu T., Yuan Q., Wang H., Wang H., Ding F., Xie X. and Jiang M. Synthesis of large single-crystal hexagonal boron nitride grains on $\mathrm{Cu}-\mathrm{Ni}$ alloy. Nature Communications, 2015. 6: 6160.

17. Wang M., Kim M., Odkhuu D., Lee J., Jang W.J., Kahng S.J., Park N., Ruoff R.S., Song Y.J. and Lee S. Catalytic transparency of hexagonal boron nitride on copper for chemical vapor deposition growth of large-area and high-quality graphene. ACS Nano, 2014. 8(6): 5478-83.

18. Kim G., Jang A.R., Jeong H.Y., Lee Z., Kang D.J. and Shin H.S. Growth of HighCrystalline, Single-Layer Hexagonal Boron Nitride on Recyclable Platinum Foil. Nano Letters, 2013. 13(4): 1834-1839.

19. Bayer B.C., Caneva S., Pennycook T.J., Kotakoski J., Mangler C., Hofmann S. and Meyer J.C. Introducing Overlapping Grain Boundaries in Chemical Vapor Deposited Hexagonal Boron Nitride Monolayer Films. Acs Nano, 2017. 11(5): 4521-4527.

20. Wang H., Zhao Y., Xie Y., Ma X. and Zhang X. Recent progress in synthesis of twodimensional hexagonal boron nitride. Journal of Semiconductors, 2017. 38(3): 031003.

21. Watanabe K., Taniguchi T. and Kanda H. Ultraviolet luminescence spectra of boron nitride single crystals grown under high pressure and high temperature. physica status solidi (a), 2004. 201(11): 2561-2565. 
22. Yuan S., Linas S., Journet C., Steyer P., Garnier V., Bonnefont G., Brioude A. and Toury B. Pure \&crystallized 2D Boron Nitride sheets synthesized via a novel process coupling both PDCs and SPS methods. Sci Rep, 2016. 6: 20388.

23. Schue L., Berini B., Betz A.C., Placais B., Ducastelle F., Barjon J. and Loiseau A. Dimensionality effects on the luminescence properties of hBN. Nanoscale, 2016. 8(13): 6986-93.

24. Schue L., Stenger I., Fossard F., Loiseau A. and Barjon J. Characterization methods dedicated to nanometer-thick hBN layers. 2D Materials, 2017. 4(1): 11.

25. Bernardo E., Ponsot I., Colombo P., Grasso S., Porwal H. and Reece M.J. Polymerderived $\mathrm{SiC}$ ceramics from polycarbosilane/boron mixtures densified by SPS. Ceramics International, 2014. 40(9, Part A): 14493-14500.

26. Esfehanian M., Oberacker R., Fett T. and Hoffmann M.J. Development of Dense FillerFree Polymer-Derived SiOC Ceramics by Field-Assisted Sintering. Journal of the American Ceramic Society, 2008. 91(11): 3803-3805.

27. Wan J., Duan R. and Mukherjee A. Spark plasma sintering of silicon nitride/silicon carbide nanocomposites with reduced additive amounts. Scripta Materialia, 2005. 53(6): 663-667.

28. Bechelany M.C., Salameh C., Viard A., Guichaoua L., Rossignol F., Chartier T., Bernard $\mathrm{S}$. and Miele P. Preparation of polymer-derived $\mathrm{Si}-\mathrm{B}-\mathrm{C}-\mathrm{N}$ monoliths by spark plasma sintering technique. Journal of the European Ceramic Society, 2015. 35(5): 1361-1374.

29. Yuan S., Toury B., Journet C. and Brioude A. Synthesis of hexagonal boron nitride graphene-like few layers. Nanoscale, 2014. 6(14): 7838-7841.

30. Yuan S., Toury B., Benayoun S., Chiriac R., Gombault F., Journet C. and Brioude A. Low-Temperature Synthesis of Highly Crystallized Hexagonal Boron Nitride Sheets with Li3N as Additive Agent. European Journal of Inorganic Chemistry, 2014. 2014(32): 5507-5513.

31. Yuan S., Toury B. and Benayoun S. Novel chemical process for preparing h-BN solid lubricant coatings on titanium-based substrates for high temperature tribological applications. Surface and Coatings Technology, 2015. 272: 366-372.

32. Schue L., Sponza L., Plaud A., Bensalah H., Watanabe K., Taniguchi T., Ducastelle F., Loiseau A. and Barjon J. Direct and indirect excitons with high binding energies in $\mathrm{hBN}$. arXiv preprint arXiv:1803.03766, 2018.

33. Wentorf R.H. Synthesis of the Cubic Form of Boron Nitride. The Journal of Chemical Physics, 1961. 34(3): 809-812.

34. Solozhenko V.L. and Turkevich V.Z. Kinetics of cBN crystallization in the Li3N-BN system at 6.6 GPa. Diamond and Related Materials, 1998. 7(1): 43-46.

35. Vel L., Demazeau G. and Etourneau J. Cubic boron nitride: synthesis, physicochemical properties and applications. Materials Science and Engineering: B, 1991. 10(2): 149-164.

36. Yuan S., Journet C., Linas S., Garnier V., Steyer P., Benayoun S., Brioude A. and Toury B. How to Increase the h-BN Crystallinity of Microfilms and Self-Standing Nanosheets: A Review of the Different Strategies Using the PDCs Route. Crystals, 2016. 6(5): 55.

37. Hoffman T.B., Clubine B., Zhang Y., Snow K. and Edgar J.H. Optimization of Ni-Cr flux growth for hexagonal boron nitride single crystals. Journal of Crystal Growth, 2014. 393: 114-118.

38. Rumble J. CRC Handbook of Chemistry and Physics. 98th ed. 2017, Boca Raton: CRC Press. 
39. Zhigadlo N.D. Crystal growth of hexagonal boron nitride (hBN) from $\mathrm{Mg}-\mathrm{B}-\mathrm{N}$ solvent system under high pressure. Journal of Crystal Growth, 2014. 402: 308-311.

40. Taniguchi T. and Watanabe K. Synthesis of high-purity boron nitride single crystals under high pressure by using Ba-BN solvent. Journal of Crystal Growth, 2007. 303(2): 525-529.

41. Silly M.G., Jaffrennou P., Barjon J., Lauret J.S., Ducastelle F., Loiseau A., Obraztsova E., Attal-Tretout B. and Rosencher E. Luminescence properties of hexagonal boron nitride: Cathodoluminescence and photoluminescence spectroscopy measurements. Physical Review B, 2007. 75(8): 5.

42. Museur L., Feldbach E. and Kanaev A. Defect-related photoluminescence of hexagonal boron nitride. Physical Review B, 2008. 78(15): 8. 